\title{
AN ASYMPTOTIC LMPI TEST FOR CYCLOSTATIONARITY DETECTION WITH APPLICATION TO COGNITIVE RADIO
}

\author{
David Ramírez ${ }^{1}$, Peter J. Schreier ${ }^{1}$, Javier Vía ${ }^{2}$, Ignacio Santamaría ${ }^{2}$ and Louis L. Scharf ${ }^{3}$ \\ ${ }^{1}$ Signal and System Theory Group, University of Paderborn, Paderborn, Germany. \\ e-mail: \{david.ramirez,peter.schreier\}@sst.upb.de \\ ${ }^{2}$ Advanced Signal Processing Group, University of Cantabria, Santander, Spain. \\ e-mail: $\{$ jvia,nacho $\} @$ gtas.dicom.unican.es \\ ${ }^{3}$ Depts. of Mathematics and Statistics, Colorado State University, Fort Collins, USA \\ e-mail: Louis.Scharf@ColoState.edu
}

\begin{abstract}
We propose a new detector of primary users in cognitive radio networks. The main novelty of the proposed detector in comparison to most known detectors is that it is based on sound statistical principles for detecting cyclostationary signals. In particular, the proposed detector is (asymptotically) the locally most powerful invariant test, i.e. the best invariant detector for low signal-to-noise ratios. The derivation is based on two main ideas: the relationship between a scalar-valued cyclostationary signal and a vector-valued wide-sense stationary signal, and Wijsman's theorem. Moreover, using the spectral representation for the cyclostationary time series, the detector has an insightful interpretation, and implementation, as the broadband coherence between frequencies that are separated by multiples of the cycle frequency. Finally, simulations confirm that the proposed detector performs better than previous approaches.
\end{abstract}

Index Terms - Cyclostationarity, Hypothesis test, Maximal invariant, Locally most powerful invariant test (LMPIT), Toeplitz matrices.

\section{INTRODUCTION}

Cognitive radio (CR) is one of the most promising technologies to improve the spectrum usage [1]. Basically, in CR the cognitive users (secondary users) are allowed to access the wireless channel, provided that the rightful owners of the spectrum (primary users) are not using it. Hence, spectrum sensing (the detection of spectral holes) is a key ingredient in CR. There are many properties that could be exploited to detect such unused frequency bands. For instance, detectors have been proposed based on the presence of known pilots, energy detection, or exploiting the spatial structure provided by multi-antenna receivers (see [2] and references therein).

In this work we exploit cyclostationary properties, which are present in most communication signals [3]. This idea is not new [2, 4], and there exist some detectors that exploit cyclostationarity in the context of CR [5-8]. Most of these detectors are based on the ideas presented in [9. 11], which basically test whether or not the cyclic covariance function or the cyclic power spectral density are zero. Nonetheless, most of these detectors are ad-hoc, but imaginative.

Our approach exploits the relationship between a scalarvalued cyclostationary signal and a vector-valued wide-sense stationary signal to properly formulate the detection problem. This idea, proposed in [12] and already used in [13] to derive the generalized likelihood ratio test (GLRT), allows us to cast the detection problem as a test for the covariance structure of the observations. Using Wijsman's theorem [14-16], in this paper we obtain the locally most powerful invariant test (LMPIT), which is the best invariant detector for low signalto-noise ratios. The use of Wijsman's theorem provides an alternative way to derive the LMPIT without the need for obtaining the maximal invariant statistic.

\section{PROBLEM FORMULATION}

In this section we consider the problem of detecting a primary user (PU) at a single-antenna cognitive user (CU). We will exploit the fact that most communications signals are cyclostationary [3], whereas the noise is wide-sense stationary (WSS). This leads to the following detection problem:

$$
\begin{aligned}
& \mathcal{H}_{1}: u[n]=(h * s)[n]+w[n], \\
& \mathcal{H}_{0}: u[n]=w[n],
\end{aligned}
$$

where $w[n]$ is the additive WSS noise, $h[n]$ is the impulse response of the wireless channel between the PU and the CU, and $s[n]$ is the transmitted signal, which is assumed to be second-order cyclostationary with known cycle period $P$.

Considering a set of $N P$ samples of $u[n]$, which are collected in the vector

$$
\mathbf{y}=\left[\begin{array}{llll}
u[0] & u[1] & \cdots & u[N P-1]
\end{array}\right]^{T} \in \mathbb{C}^{N P},
$$


we will formulate the detection problem as a test for the covariance structure of $\mathbf{y}$, which we will now determine.

Under $\mathcal{H}_{0}$, it is clear that the covariance matrix of $\mathbf{y}$ is

$$
\mathbf{R}_{0}=\left[\begin{array}{cccc}
r[0] & r[-1] & \cdots & r[-N P+1] \\
r[1] & r[0] & \cdots & r[-N P+2] \\
\vdots & \vdots & \ddots & \vdots \\
r[N P-1] & r[N P-2] & \cdots & r[0]
\end{array}\right],
$$

where $r[m]=E\left[u[n] u^{*}[n-m]\right]$ is the correlation sequence of $u[n]$ under $\mathcal{H}_{0}$, the complex conjugate is denoted by $*$, and the covariance matrix $\mathbf{R}_{0}$ is a Hermitian Toeplitz matrix. Under $\mathcal{H}_{1}$ the derivation is a bit more involved. Following [13], we reshape $u[n]$ into blocks of size $P$ to obtain the vectorvalued time series

$$
\mathbf{x}[n]=\left[\begin{array}{lll}
u[n P] & \cdots & u[(n+1) P-1
\end{array}\right]^{T},
$$

which is WSS [12] with matrix-valued covariance sequence $\mathbf{Q}[m]=E\left[\mathbf{x}[n] \mathbf{x}^{H}[n-m]\right] \in \mathbb{C}^{P \times P}$. Stacking $N$ of these vectors, $\mathbf{x}[0], \ldots, \mathbf{x}[N-1]$, the vector $\mathbf{y}$ may be rewritten as

$$
\mathbf{y}=\left[\begin{array}{llll}
\mathbf{x}^{T}[0] & \mathbf{x}^{T}[1] & \cdots & \mathbf{x}^{T}[N-1]
\end{array}\right]^{T} .
$$

Hence, the covariance matrix of $\mathbf{y}$ under $\mathcal{H}_{1}$ is

$$
\mathbf{R}_{1}=\left[\begin{array}{cccc}
\mathbf{Q}[0] & \mathbf{Q}[-1] & \cdots & \mathbf{Q}[-N+1] \\
\mathbf{Q}[1] & \mathbf{Q}[0] & \cdots & \mathbf{Q}[-N+2] \\
\vdots & \vdots & \ddots & \vdots \\
\mathbf{Q}[N-1] & \mathbf{Q}[N-2] & \cdots & \mathbf{Q}[0]
\end{array}\right]
$$

which is a Hermitian block-Toeplitz matrix with block size $P$.

Finally, assuming that the transmitted signal and the noise are Gaussian, the test in (1) becomes

$$
\begin{aligned}
& \mathcal{H}_{1}: \mathbf{y} \sim \mathcal{C N}\left(\mathbf{0}, \mathbf{R}_{1}\right), \\
& \mathcal{H}_{0}: \mathbf{y} \sim \mathcal{C N}\left(\mathbf{0}, \mathbf{R}_{0}\right)
\end{aligned}
$$

We are thus testing whether the covariance matrix of $\mathbf{y}$ is Toeplitz or block-Toeplitz with known block-size $P$. This hypothesis testing problem is invariant to the transformation group $\mathcal{G}=\{g: \mathbf{y} \rightarrow g(\mathbf{y})=\alpha \mathbf{y}\}$, which reparametrizes the complex normal distributions as

$$
\overline{\mathcal{G}}=\left\{\bar{g}:\left(\mathbf{R}_{1}, \mathbf{R}_{0}\right) \rightarrow \bar{g}\left(\mathbf{R}_{1}, \mathbf{R}_{0}\right)=\left(|\alpha|^{2} \mathbf{R}_{1},|\alpha|^{2} \mathbf{R}_{0}\right)\right\}
$$

\section{DERIVATION OF THE LMPIT}

The derivation of the uniformly most powerful invariant test (UMPIT) and the LMPIT usually requires the maximal invariant statistic and its distribution under both hypotheses [17]. With a few exceptions, deriving the distributions of the maximal invariant statistic is extremely difficult or even impossible. As an alternative approach, in this work, we use Wijsman's theorem [14,15], which allows us to obtain the ratio of the distributions of the maximal invariant statistic without deriving such distributions or even the maximal invariant statistic. Wijsman's theorem states that the ratio of the distributions of the maximal invariant statistic may be expressed, under some mild conditions [18,19], as

$$
\mathscr{L}=\frac{\int_{\mathcal{G}} p\left(g(\mathbf{y}) ; \mathcal{H}_{1}\right)\left|\operatorname{det}\left(\mathbf{J}_{g}\right)\right| d g}{\int_{\mathcal{G}} p\left(g(\mathbf{y}) ; \mathcal{H}_{0}\right)\left|\operatorname{det}\left(\mathbf{J}_{g}\right)\right| d g}
$$

where $p\left(g(\mathbf{y}) ; \mathcal{H}_{i}\right)$ denotes the probability density function of the transformed observations under $\mathcal{H}_{i}, \mathbf{J}_{g}$ is the Jacobian of the transformation $g(\cdot)$ and $d g$ is an invariant group measure, which may be taken as the usual Lebesgue measure. However, due to the invariances of (2), the UMPIT (or LMPIT) does not exist. We must therefore first rewrite the problem by considering the asymptotic case of $N \rightarrow \infty$.

It is well known that as $N \rightarrow \infty$, block-Toeplitz (and Toeplitz) matrices are equivalent to block-circulant (and circulant) matrices [20,21]. Moreover, it was proved in [22, 23] that the likelihood with a block-Toeplitz (or Toeplitz) covariance matrix converges in the mean-square sense to the likelihood with a block-circulant (or circulant) covariance matrix. This allows us to replace the covariance matrices in (2) by circulant approximations:

$$
\begin{aligned}
& \mathbf{R}_{1} \approx\left(\mathbf{F}_{N} \otimes \mathbf{I}_{P}\right) \mathbf{V}_{\mathrm{B}}\left(\mathbf{F}_{N} \otimes \mathbf{I}_{P}\right)^{H} \\
& \mathbf{R}_{0} \approx \mathbf{F}_{N P} \mathbf{V}_{\mathrm{D}} \mathbf{F}_{N P}^{H} .
\end{aligned}
$$

Here, $\mathbf{F}_{N}$ is the discrete Fourier transform (DFT) matrix of dimension $N \times N, \mathbf{V}_{\mathrm{D}}$ is a diagonal matrix that contains the power spectral density (PSD) under $\mathcal{H}_{0}$, i.e. the $N P$ points DFT of $\{r[m]\}_{m=0}^{N P-1}$, and $\mathbf{V}_{\mathrm{B}}$ is a block-diagonal matrix that contains the PSD matrix under $\mathcal{H}_{1}$, that is, the $N$ points DFT of $\{\mathbf{Q}[m]\}_{m=0}^{N-1}$. Let us now transform the data as $\mathbf{z}=\mathbf{L}_{N P, N} \mathbf{F}_{N P}^{H} \mathbf{y}$, where $\mathbf{L}_{N P, N}$ is the commutation (or stride permutation) matrix ${ }^{1}$ This transformation basically obtains the DFT of the sequence $\{u[n]\}_{n=0}^{N P-1}$ and rearranges the frequencies, which yields

$$
\begin{aligned}
& \mathcal{H}_{1}: \mathbf{z} \sim \mathcal{C N}\left(\mathbf{0}, \mathbf{S}_{\mathrm{B}}\right), \\
& \mathcal{H}_{0}: \mathbf{z} \sim \mathcal{C N}\left(\mathbf{0}, \mathbf{S}_{\mathrm{D}}\right),
\end{aligned}
$$

where $\mathbf{S}_{B}$ is a positive-definite block-diagonal matrix and $\mathbf{S}_{D}$ is diagonal with nongengative entries. These covariance matrices are linearly related to $V_{B}$ and $V_{D}$. Due to lack of space, we omit the details, which will be presented in a forthcoming journal version of this paper.

To apply Wijsman's theorem to (3) we need the group of invariant transformations. We may only consider linear transformations since we must preserve Gaussianity. Moreover, it is clear that a multiplication by a diagonal matrix (equivalent to linear filtering of $u[n]$ ) does not modify the structure of the covariance matrices. Finally, the structure is also preserved

\footnotetext{
${ }^{1}$ The commutation matrix fulfils $\operatorname{vec}(\mathbf{A})=\mathbf{L}_{N P, N} \operatorname{vec}\left(\mathbf{A}^{T}\right)$, where $\mathbf{A}$ is a $P \times N$ matrix.
} 


$$
\mathscr{L}=\frac{\sum_{\mathbb{P}_{N}, \mathbb{P}_{P}} \int_{\mathbb{D}} \operatorname{det}\left(\mathbf{S}_{\mathrm{B}}\right)^{-M}\left|\operatorname{det}(\mathbf{G})^{2 M}\right| \exp \left[-M \operatorname{tr}\left(\mathbf{S}_{\mathrm{B}}^{-1} \mathbf{P G} \hat{\mathbf{S}} \mathbf{G}^{*} \mathbf{P}^{T}\right)\right] d \mathbf{G}}{\sum_{\mathbb{P}_{N}, \mathbb{P}_{P}} \int_{\mathbb{D}} \operatorname{det}\left(\mathbf{S}_{\mathrm{D}}\right)^{-M}\left|\operatorname{det}(\mathbf{G})^{2 M}\right| \exp \left[-M \operatorname{tr}\left(\mathbf{S}_{\mathrm{D}}^{-1} \mathbf{P} \mathbf{G} \hat{\mathbf{S}} \mathbf{G}^{*} \mathbf{P}^{T}\right)\right] d \mathbf{G}},
$$

by a matrix that permutes the blocks of $\mathbf{S}_{\mathrm{B}}$, and also by a matrix that permutes the elements within each block of $\mathbf{S}_{\mathrm{B}}$. Hence, the group of invariant transformations is

$$
\mathcal{G}=\left\{g: \mathbf{z} \rightarrow g(\mathbf{z})=\mathbf{P G z}, \mathbf{P}=\mathbf{P}_{N} \otimes \mathbf{P}_{P}\right\},
$$

where $\mathbf{P}_{N} \in \mathbb{P}_{N}, \mathbf{G} \in \mathbb{D}, \mathbb{P}_{N}$ is the set of $N$-dimensional permutation matrices, and $\mathbb{D}$ is the set of invertible diagonal matrices. Assuming $M$ independent and identically distributed realizations of $\mathbf{z}$, the ratio of the distributions of the maximal invariant statistic is presented in (4) on top of this page. In this equation we have summed over all possible permutations since the permutation group is a finite group, and the sample PSD matrix is

$$
\hat{\mathbf{S}}=\frac{1}{M} \sum_{n=0}^{M-1} \mathbf{z}_{n} \mathbf{z}_{n}^{H}
$$

In the following, we will show that there does not exist a UMPIT for the detection problem, although it is possible to find the LMPIT. Again, due to the lack of space, the proofs will be presented in the journal version.

Lemma 1 The ratio of the distributions of the maximal invariant statistic may be simplified as

$$
\mathscr{L} \propto \sum_{\mathbb{P}_{N}, \mathbb{P}_{P}} \int_{\mathbb{D}}\left|\operatorname{det}(\mathbf{G})^{2 M}\right| e^{-\alpha} d \mathbf{G}
$$

where

$$
\alpha=M \sum_{k=1}^{N} \operatorname{tr}\left(\tilde{\mathbf{S}}_{k} \mathbf{G}_{k} \hat{\mathbf{C}}_{k} \mathbf{G}_{k}^{*}\right),
$$

the coherence matrix is $\hat{\mathbf{C}}=\hat{\mathbf{D}}^{-1 / 2} \hat{\mathbf{S}} \hat{\mathbf{D}}^{-1 / 2}$ with $\hat{\mathbf{D}}=$ $\operatorname{diag}(\hat{\mathbf{S}})$. Here $\mathbf{G}_{k}$ is the $k$ th $P \times P$ block on the diagonal of $\mathbf{G}$, which is also diagonal, $\hat{\mathbf{C}}_{k}$ is the $k$ th $P \times P$ block on the diagonal of $\hat{\mathbf{C}}$, and $\tilde{\mathbf{S}}_{k}$ is a permutation of the kth block on the diagonal of dimensions $P \times P$ of the whitened version of $\mathbf{S}_{\mathrm{B}}^{-1}$, i.e. of $\operatorname{diag}\left(\mathbf{S}_{\mathrm{B}}^{1 / 2}\right) \mathbf{S}_{\mathrm{B}}^{-1} \operatorname{diag}\left(\mathbf{S}_{\mathrm{B}}^{1 / 2}\right)$.

From Lemma 1 it is clear that $\mathscr{L}$ does not depend on the diagonal elements of $\hat{\mathbf{S}}$, which are the estimated PSD. Hence, the detector will not depend on the PSD, but on a normalized cyclic PSD, i.e. a coherence. Moreover, $\mathscr{L}$ is a function of the unknown parameters, namely $\mathbf{S}_{\mathrm{B}}$, and the UMPIT does therefore not exist. However, as previously pointed out, it is still possible to derive the LMPIT. To do so, let us consider the case of close hypotheses, i.e. low signal-to-noise ratio (SNR), in which $\mathbf{S}_{\mathrm{B}}$ is approximately diagonal, and therefore the whitened covariance matrix is $\tilde{\mathbf{S}}_{\mathrm{B}} \approx \mathbf{I}$. Thus, using the second-order Taylor series of $e^{-\alpha}$, the ratio $\mathscr{L}$ becomes

$$
\mathscr{L} \propto \sum_{\mathbb{P}_{N}, \mathbb{P}_{P}} \int_{\mathbb{D}} \beta(\mathbf{G})\left[\left(\alpha-\alpha_{0}\right)^{2}-2\left(\alpha-\alpha_{0}\right)\right] d \mathbf{G},
$$

with $\alpha_{0}=M \operatorname{tr}\left(\mathbf{G G}^{*}\right)$ and $\beta(\mathbf{G})=\left|\operatorname{det}(\mathbf{G})^{2 M}\right| e^{-M \operatorname{tr}\left(\mathbf{G G}^{*}\right)}$. Finally, after some tedious algebra, we obtain the asymptotic LMPIT, which is presented in the following theorem.

Theorem 1 The LMPIT accepts $\mathcal{H}_{0}$ for small values of

$$
T\left(\mathbf{y}_{0}, \ldots, \mathbf{y}_{M-1}\right)=\sum_{k=1}^{N}\left\|\hat{\mathbf{C}}_{k}\right\|^{2} .
$$

The LMPIT presented in this theorem is similar to the LMPIT presented in [7] as both LMPIT are sums of Frobenius norms of certain matrices. Yet, there are differences between our approach and [7] regarding the underlying assumptions. The main difference is that the authors of [7] considered the case of white noise. Moreover, they did not actually prove that the detector is the LMPIT.

Finally, let us present a more insightful form and interpretation for the LMPIT.

Remark 1 Let us denote by $S\left(e^{j \theta}, e^{j 2 \pi c / P}\right)$ the cyclic PSD at cycle frequency $2 \pi c / P$ and by $S\left(e^{j \theta}\right)$ the PSD. Then, the LMPIT may be written as

$$
T=\sum_{c=1}^{P-1} \sum_{k=0}^{(P-c) N-1} \frac{\left|S\left(e^{j \theta_{k}}, e^{j 2 \pi c / P}\right)\right|^{2}}{S\left(e^{j \theta_{k}}\right) S\left(e^{j\left(\theta_{k}+2 \pi c / P\right)}\right)},
$$

where $\theta_{k}=2 \pi k / N P$. Moreover, as $N \rightarrow \infty$, the LMPIT becomes

$$
T=\sum_{c=1}^{P-1} \int_{0}^{(P-c) 2 \pi / P} \frac{\left|S\left(e^{j \theta}, e^{j 2 \pi c / P}\right)\right|^{2}}{S\left(e^{j \theta}\right) S\left(e^{j(\theta+2 \pi c / P)}\right)} \frac{d \theta}{2 \pi} .
$$

Here, one comment is in order. The ratio between the cyclic PSD and the product of the PSD at frequencies separated by the cycle frequency was already proposed in [10], which, however, only used one cycle frequency and one global frequency.

Remark 2 Since the cyclic PSD may be rewritten as [24]

$$
S\left(e^{j \theta}, e^{j 2 \pi c / P}\right) \frac{d \theta}{2 \pi}=E\left[d \xi\left(e^{j \theta}\right) d \xi^{*}\left(e^{j(\theta-2 \pi c / P)}\right)\right],
$$

where $d \xi\left(e^{j \theta}\right)$ is an increment of the complex spectral process $\xi\left(e^{j \theta}\right)$ for the Loève representation [25], the LMPIT is just a function of the correlation coefficient (or coherence) between $d \xi\left(e^{j \theta}\right)$ and $d \xi\left(e^{j(\theta-2 \pi c / P)}\right)$. 


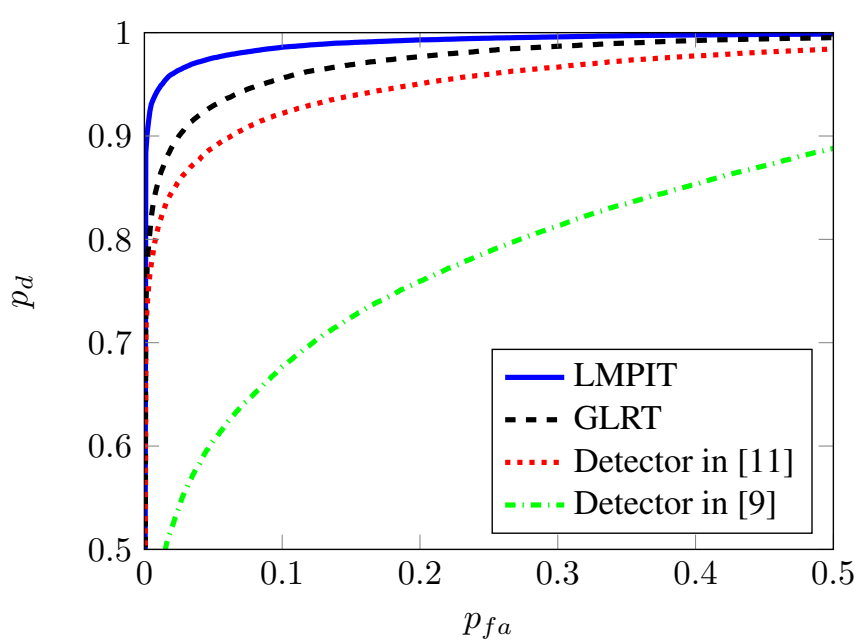

Fig. 1: ROC curve for an experiment with $P=4, M=5$ snapshots, $N=256$, and SNR $=-5 \mathrm{~dB}$

\section{NUMERICAL RESULTS}

In this section we evaluate the performance of the proposed detector and compare it to that of previous detectors. In particular, we compare our approach with those of [9.11], and with the GLRT of [13]. For [9, 11], it is necessary to specify a priori the $\operatorname{lag}(\mathrm{s})$ of the cyclic correlation function that will be used. This, of course, would require a priori knowledge about the cyclic correlation function, which is usually unknown. To make a fair comparison, for the detector in [11] we decided to only use the lags $0,1,2$ and 3 of the cyclic correlation. However, for the detector in [9], which only uses one lag of the cyclic correlation, selecting the first lag would result in poor performance (depending on the shaping filter since it determines the cyclic covariance), so we use the lag that maximizes the cyclic correlation function, although this might be unrealistic in some problems. Moreover, we apply a Kaiser window of length 1025 to estimate the cyclic PSD required for the detector in [11].

For the simulations, we simulate noise that is a moving average process of order 19 (20 coefficients at the sampling rate). The channel is Rayleigh with 40 taps (at the sampling rate) and an exponential power delay profile with delay spread $7.57 \mu \mathrm{s}$, and the transmitted signal is QPSK with rectangular shaping and a symbol rate of $300 \mathrm{Kbauds} / \mathrm{second}$. Moreover, the sampling frequency is $1.2 \mathrm{MHz}$, yielding 4 samples per symbol, which coincides with the cycle period $P=4$. We consider $M=5$ realizations of length $N=256$ symbols, so the total number of available samples is $4 \cdot 5 \cdot 256=5120$ samples. The noise and channel coefficients were randomly generated in each Monte Carlo simulation, following a Gaussian distribution.

In the first experiment, we obtain the receiver operating

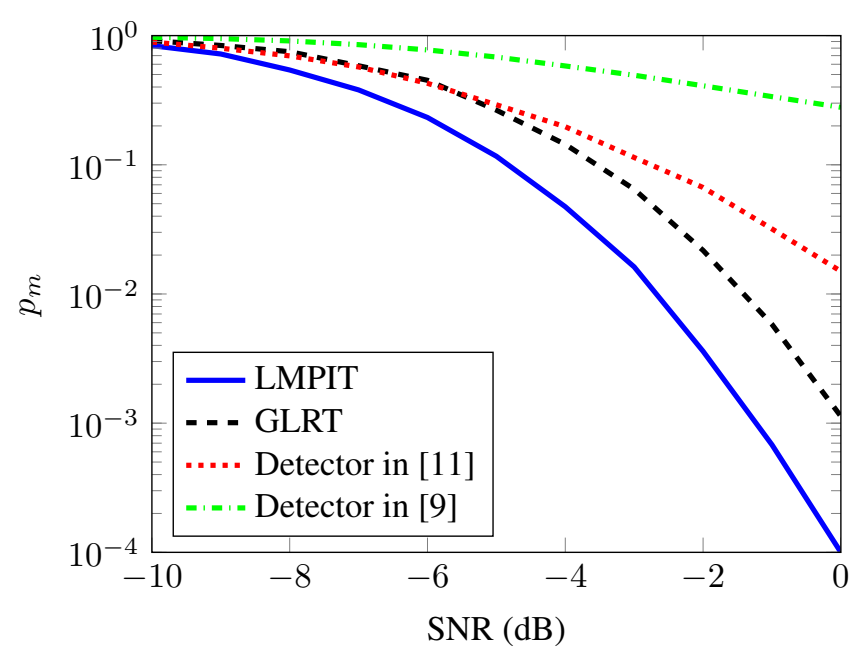

Fig. 2: Probability of missed detection vs. SNR for $p_{f a}=$ $10^{-3}$ in an experiment with $P=4, M=5$, and $N=256$

characteristic (ROC) curves of the detectors for a signal-tonoise ratio (SNR) of $-5 \mathrm{~dB}$. As can be seen in Figure 1, the LMPIT presents the best performance, even for this case of non-Gaussian signals. After the LMPIT, the best detector is the GLRT. This good behavior of both LMPIT and GLRT may be explained by the fact that they exploit the broadband coherence at all cycle frequencies and all lags, whereas the other detectors do not. Finally, Figure 2 shows the probability of missed detection vs. SNR for a fixed $p_{f a}=10^{-3}$, where we can observe a similar behavior.

\section{CONCLUSIONS}

We derived a detector for cognitive radio based on cyclostationarity. By exploiting the relationship between a scalarvalued cyclostationary time series and a vector-valued widesense stationary time series, we reformulated (asymptotically) the detection problem as a test for diagonality vs. blockdiagonality of the covariance matrix. For this problem, we derived the LMPIT using Wijsman's theorem. Numerical results show the better performance of our detector compared to previously proposed approaches.

\section{ACKNOWLEDGMENTS}

The work of P. Schreier was supported by the Alfried Krupp von Bohlen und Halbach Foundation, under its program "Return of German scientists from abroad". The work of I. Santamaría and J. Vía was supported by the Spanish Government, Ministerio de Ciencia e Innovación (MICINN), under project RACHEL (TEC2013-47141-C4-3-R). The work of L. Scharf was supported by the Airforce Office of Scientific Research under contract FA9550-10-1-0241. 


\section{REFERENCES}

[1] J. M. Peha, "Sharing spectrum through spectrum policy reform and cognitive radio," Proc. IEEE, vol. 97, no. 4, pp. 708-719, Apr. 2009.

[2] E. Axell, G. Leus, E.G. Larsson, and H.V. Poor, "Spectrum sensing for cognitive radio : State-of-the-art and recent advances," IEEE Signal Process. Mag., vol. 29, no. 3, pp. 101-116, May 2012.

[3] W. A. Gardner, W. Brown, and Chih-Kang Chen, "Spectral correlation of modulated signals: Part II-digital modulation," IEEE Trans. Comm., vol. 35, no. 6, pp. 595-601, Jun 1987.

[4] Y. Zeng, Y.-C. Liang, A. T. Hoang, and R. Zhang, "A review on spectrum sensing for cognitive radio: Challenges and solutions," EURASIP Journal Advances Signal Process., 2010.

[5] E. Axell and E. G. Larsson, "Multiantenna spectrum sensing of a second-order cyclostationary signal," in IEEE Int. Work. Comp. Adv. in Multi-Sensor Adaptive Process., Dec. 2011, pp. 329-332.

[6] J. Lundén, V. Koivunen, A. Huttunen, and H. V. Poor, "Collaborative cyclostationary spectrum sensing for cognitive radio systems," IEEE Trans. Signal Process., vol. 57, no. 11, pp. 4182-4195, Nov. 2009.

[7] J. Riba, J. Font-Segura, J. Villares, and G. Vazquez, "Frequency-domain GLR detection of a second-order cyclostationary signal over fading channels," IEEE Trans. Signal Process., vol. 62, no. 8, pp. 1899-1912, Apr. 2014.

[8] P. Urriza, E. Rebeiz, and D. Cabric, "Multiple antenna cyclostationary spectrum sensing based on the cyclic correlation significance test," IEEE Journal on Selected Areas in Communications, vol. 31, no. 11, pp. 21852195, November 2013.

[9] S.V. Schell and W.A. Gardner, "Detection of the number of cyclostationary signals in unknown interference and noise," in Asilomar Conf. on Signals, Systems and Computers, Nov. 1990, vol. 1, p. 473.

[10] S. Enserink and D. Cochran, "On detection of cyclostationary signals," in Proc. IEEE Int. Conf. Acoust., Speech and Signal Process. (ICASSP), Detroit, USA, May 1995, pp. 2004-2007.

[11] A. V. Dandawaté and G. B. Giannakis, "Statistical tests for presence of cyclostationarity," IEEE Trans. Signal Process., vol. 42, no. 9, pp. 2355-2369, Sep. 1994.

[12] E. D. Gladyshev, "Periodically correlated random sequences," Soviet Math. Dokl., vol. 2, pp. 385-388, 1961.
[13] D. Ramírez, L. L. Scharf, J. Vía, I. Santamaría, and P. J. Schreier, "An asymptotic GLRT for the detection of cyclostationary signals," in IEEE Int. Conf. on Acoustics, Speech and Signal Process., Florence, Italy, May 2014.

[14] R. A. Wijsman, "Cross-sections of orbits and their application to densities of maximal invariants," in Proc. Fifth Berkeley Symp. on Math. Stat. and Prob., 1967, vol. 1, pp. 389-400.

[15] J. R. Gabriel and S. M. Kay, “Use of Wijsman's theorem for the ratio of maximal invariant densities in signal detection applications," in Asilomar Conf. Signals, Systems and Computers, Nov. 2002, vol. 1, pp. 756 - 762.

[16] D. Ramírez, J. Vía, I. Santamaría, and L. L. Scharf, "Locally most powerful invariant tests for correlation and sphericity of Gaussian vectors," IEEE Trans. Inf. Theory, vol. 59, no. 4, pp. 2128-2141, Apr. 2013.

[17] L. L. Scharf, Statistical Signal Processing: Detection, Estimation, and Time Series Analysis, Addison - Wesley, 1991.

[18] R. A. Wijsman, "Proper action in steps, with application to density ratios of maximal invariants," The Annals of Statistics, vol. 13, no. 1, pp. 395-402, 1985.

[19] R. A. Wijsman, "Correction: Proper action in steps, with application to density ratios of maximal invariants," The Annals of Statistics, vol. 21, no. 4, pp. 2168-2169, Dec. 1993.

[20] R. M. Gray, Toeplitz and Circulant Matrices: A Review, Foundations and Trends in Communications and Information Theory, 2006.

[21] J. Gutiérrez-Gutiérrez and P. M. Crespo, “Asymptotically equivalent sequences of matrices and Hermitian block Toeplitz matrices with continuous symbols: Applications to MIMO systems," IEEE Trans. Inf. Theory, vol. 54, no. 12, pp. 5671-5680, Dec. 2008.

[22] D. Ramírez, J. Vía, I. Santamaría, and L. L. Scharf, "Detection of spatially correlated Gaussian time series," IEEE Trans. Signal Process., vol. 58, no. 10, Oct. 2010.

[23] D. Ramírez, G. Vázquez-Vilar, R. López-Valcarce, J. Vía, and I. Santamaría, "Detection of rank- $P$ signals in cognitive radio networks with uncalibrated multiple antennas," IEEE Trans. Signal Process., vol. 59, no. 8, pp. 3764-3774, Aug. 2011.

[24] P. J. Schreier and L. L. Scharf, Statistical Signal Processing of Complex-Valued Data, Cambridge University Press, 2010.

[25] M. Loève, Probability Theory II, New York: Springer, 4th edition, 1978. 